\title{
Verification Conditions Are Code
}

\author{
Andrew M Gravell
}

May 3, 2006

\begin{abstract}
This paper presents a new theoretical result concerning Hoare Logic. It is shown here that the verification conditions which support a Hoare Logic program derivation are themselves sufficient to construct a correct implementation of the given pre-, post- condition specification. This property is mainly of theoretical interest, though it is possible that it may have some practical use, for example if predicative programming methodology is adopted. The result is shown to hold for both the original, partial correctness, Hoare logic, and also a variant for total correctness derivations.
\end{abstract}

\section{Introduction}

\subsection{Background and Motivation}

Hoare logic, and variants thereof, have been in use since Hoare's original paper [20]. In this formal system, a correctness proof typically depends on some verification conditions of the form $\mathrm{A} \Rightarrow \mathrm{B}$. These must themselves be proved, using some system of predicate calculus, in order to show that the Hoare logic derivation is correct. The result here concerns the verification conditions that support a given Hoare logic derivation. Surprisingly, these alone (together with the final pre- and post-condition) are sufficient to allow a proof of a correct implementation to be constructed.

Let us motivate this result by giving an example. Consider the following program [27] to calculate the integer square root of a natural number $s$.

$$
\begin{aligned}
& \{s \geq 0\} \\
& q, r:=s+1,0 \\
& \text { while } r+1 \neq q \text { do } \\
& \quad p:=(q+r) \div 2 \\
& \quad \text { if } s<p^{2} \text { then } q:=p \text { else } r:=p \text { fi } \\
& \text { end } \\
& \left\{r^{2} \leq s<(r+1)^{2}\right\}
\end{aligned}
$$

The correctness of this program depends on four verification conditions

$$
\begin{aligned}
& s \geq 0 \Rightarrow 0^{2} \leq s<(s+1)^{2} \\
& r^{2} \leq s<q^{2} \wedge(r+1 \neq q) \Rightarrow r^{2} \leq s<(r+1)^{2} \\
& r^{2} \leq s<q^{2} \wedge r+1 \neq q \leq s<p^{2} \Rightarrow r^{2} \leq s<p^{2} \\
& r^{2} \leq s<q^{2} \wedge r+1 \neq q \wedge\left(s<p^{2}\right) \Rightarrow p^{2} \leq s<q^{2}
\end{aligned}
$$


Given these conditions, the assignment statements can be derived by finding substitutions that convert one clause to another. These can be written as Hoare triples such as

and

$$
\left\{0^{2} \leq s<(s+1)^{2}\right\} \quad q, r:=s+1,0\left\{r^{2} \leq s<q^{2}\right\}
$$

$$
\left\{r^{2} \leq s<p^{2}\right\} q:=p\left\{r^{2} \leq s<q^{2}\right\}
$$

These Hoare triples can then be combined using the laws of Hoare logic to construct the correctness argument, and hence the original program itself. Thus the verification conditions can be said to contain the essence of the correctness argument.

The main result of this paper will be to show that there is an algorithm to construct this program and its proof given as input the pre-, post-, and verification conditions. Alternatively, if the given conditions do not form the basis of a Hoare Logic derivation, the algorithm will terminate and report that no such program exists. That is to say, it is a decision procedure.

Of course, there are other partially correct programs also based on the same verification conditions. In particular

$$
\begin{aligned}
& \{s \leq 0\} \\
& q, r:=s+1,0 \\
& \text { while } r+1 \neq q \text { do } \\
& \quad \text { if } s<p^{2} \text { then } q:=p \text { else } r:=p \text { fi } \\
& \text { end } \\
& \left\{r^{2} \leq s<(r+1)^{2}\right\}
\end{aligned}
$$

is a valid Hoare triple. That is to say, the assignment to $p$ is redundant when considering only partial correctness.

The algorithm described here will search for an implementation that is in some sense minimal, using only necessary assignments. There is also a variant of the algorithm deals that with total correctness.

A particular appeal of Hoare Logic is its simplicity. Systems with few rules are most amenable to proof theoretic approaches, which typically require structural induction (or equivalent) with at least one case per axiom or rule. The system studied here is the original Hoare logic [20]. Various extensions of this have been published to cover programming language features such as procedures [21] and also including an axiomatic semantics for the Pascal programming language [22].

More recently, formal methods researchers have developed refinement calculi $[4,28,27]$. These are more suitable for top-down program derivation, with more laws so that derivations can be abbreviated, which means they are not as well suited to the proof theoretic methods used here. In general, also, refinement calculus researchers [5] have adopted a model-theoretic approach, focussing on semantics, not axioms. The relationship between Hoare Logic and refinement calculus is explained by [1], and a unifying framework for both these systems (and others) is presented by [24].

Note that as this paper is concerned with Hoare Logic, the word "proof" usually refers to a Hoare logic derivation of a valid Hoare triple. 


\subsection{Notation and Definitions}

For simplicity, it is assumed here that all programming language tests used in if and while statements are (syntactically identical to) logical predicates (though not necessarily vice versa). Similarly, no distinction is made between assignments statements and substitutions. Issues such as undefined expressions and type correctness are not considered here. To avoid syntactic ambiguities such as the dangling else, the keyword fi is hereafter used to terminate each if statement, and end to terminate each while statement.

Other notations and notational conventions used in this paper are:

$\alpha, \beta, \gamma, \alpha^{\prime} \quad$ assignment statements

$\mathrm{A}, \mathrm{B}, \mathrm{C}, \mathrm{A}^{\prime}$ predicates/assertions/conditions

$\alpha(\mathrm{A}) \quad$ syntactic substitution

$\mathrm{P}, \mathrm{Q}, \mathrm{R}, \mathrm{P}^{\prime} \quad$ programs and sub-programs

A P B, C Q D Hoare triples (cf. the conventional $\{\mathrm{A}\} \mathrm{P}\{\mathrm{B}\}$ etc.)

$\mathrm{V}, \mathrm{V}^{\prime}, \mathrm{W}$ sets of conditions

Note that since sets of conditions are not typically nested it is convenient to omit the usual braces. For example if $\mathrm{V}=\mathrm{A}, \mathrm{B}$ and $\mathrm{V}^{\prime}=\mathrm{V}, \mathrm{C}$ then $\mathrm{V}^{\prime}=\mathrm{A}, \mathrm{B}, \mathrm{C}$. Finally note that, although unconventional, omitting the braces from Hoare triples simplifies and abbreviates the later presentation.

In this paper it is assumed that substitutions can be multiple (also known as parallel assignments) [15]. For example $x, y:=y, x$ is a classic multiple assignment that swaps the values of variables $x$ and $y$.

The classical form of Hoare logic, using these notational conventions, is given in Figure 1.

$$
\begin{aligned}
& \vdash \alpha(\mathrm{A}) \alpha \mathrm{A} \\
& \mathrm{A} \Rightarrow \mathrm{B}, \mathrm{B} \mathrm{P} \mathrm{C} \vdash \mathrm{A} \mathrm{P} \mathrm{C} \quad \text { consequence: pre-condition } \\
& \mathrm{A} \mathrm{P} \mathrm{B}, \mathrm{B} \Rightarrow \mathrm{C} \vdash \mathrm{A} \mathrm{P} \mathrm{C} \text { consequence: post-condition } \\
& \text { A P B, B Q C } \vdash \text { A P Q C sequence } \\
& \mathrm{A} \wedge \mathrm{B} \mathrm{P} \mathrm{C}, \mathrm{A} \wedge \neg \mathrm{B} \mathrm{Q} \mathrm{C} \vdash \mathrm{A} \text { if } \mathrm{B} \text { then } \mathrm{P} \text { else } \mathrm{Q} \text { fi } \mathrm{C} \\
& \text { selection } \\
& \mathrm{A} \wedge \mathrm{B} \mathrm{P} \mathrm{A} \vdash \mathrm{A} \text { while } \mathrm{B} \text { do } \mathrm{P} \text { end } \mathrm{A} \wedge \neg \mathrm{B} \\
& \text { iteration }
\end{aligned}
$$

Figure 1: Classical Hoare Logic

\subsection{Basic Properties of Classical Hoare Logic}

Some basic properties of classical Hoare logic are now presented.

Lemma 1 For any programs P, Q, R, conditions A, B, the Hoare triple A $\mathrm{P} ;(\mathrm{Q} ; \mathrm{R}) \mathrm{B}$ is valid if and only if $\mathrm{A}(\mathrm{P} ; \mathrm{Q}) ; \mathrm{R} \mathrm{B}$ is.

Proof 
A $\mathrm{P} ;(\mathrm{Q} ; \mathrm{R}) \mathrm{B}$ is valid iff

A P C and C Q;R B for some condition $\mathrm{C}$, iff

A P C and C Q D and D R B for some conditions C, D, iff

A P;Q D and D R B for some condition D, iff

A (P:Q);R B

Note that this property is very familiar from the study of program semantics, for example in the theory of predicate transformers, where this result would follow directly from the associativity of function composition. Working directly with program semantics is a model-theoretic approach, however, in contrast to the proof-theoretic approach adopted here. Where a property such as the one above can be expressed both model- and proof-theoretically, it is typically the case that the property can be established more easily using model-theoretic techniques. The main theorem presented here, however, concerns verification conditions, which have no equivalent in standard program semantics, so the proof-theoretic approach is more natural.

An important property of Hoare Logic is the substitution principle. This is also well-known in programming logics and semantics.

Lemma 2 (Substitutability) Suppose programs P and Q are such that for any conditions A and B, A P B is a valid Hoare triple whenever if A Q B is. Then program $\mathrm{P}$ can validly replace program $\mathrm{Q}$ in any Hoare proof.

Proof The Hoare triple A Q B must appear in the Hoare proof at some point. The Hoare triple A P B must also be valid by the hypothesis. Thereafter, any proof steps that involve the use of $\mathrm{Q}$ as a sub-program (via the use of the sequence, conditional or iteration law) can be replaced by the equivalent proof step, but with $\mathrm{P}$ replacing $\mathrm{Q}$. This follows from the fact that the sequence, conditional, and iteration laws constrain only the conditions occurring as their hypotheses, not the programs.

The model-theoretic equivalent of this substitution principle is classically called monotonicity of program refinement. For example, combining lemmas 1 and 2, the programs $(\mathrm{P} ; \mathrm{Q}) ; \mathrm{R}$ and $\mathrm{P} ;(\mathrm{Q} ; \mathrm{R})$ can freely replace each other in any Hoare logic derivation. Given this result, and the conventions given above, it is therefore safe to omit semi-colons altogether. For example, P Q is an abbreviation for the sequence P; Q. Similarly, P Q R abbreviates the program $(\mathrm{P} ; \mathrm{Q}) ; \mathrm{R}$ which as noted above is essentially equivalent to $\mathrm{P} ;(\mathrm{Q} ; \mathrm{R})$.

Definition: a (Hoare) proof is based on a set of predicates $\mathrm{V}$ if and only if every instance of the rules of consequence (e.g. $\mathrm{A} \Rightarrow \mathrm{B}, \mathrm{B} \mathrm{P} \mathrm{C} \vdash \mathrm{A}$ P C) in the proof uses a predicate (here $\mathrm{A} \Rightarrow \mathrm{B}$ ) that is a member of $\mathrm{V}$.

Definition: if $\mathrm{P}$ can replace $\mathrm{Q}$ and $\mathrm{Q}$ can replace $\mathrm{P}$ in any proof, and both are based on the same verification conditions, then $\mathrm{P}$ and $\mathrm{Q}$ are said to be proof-equivalent.

Lemma 3 if $\mathrm{C}$ then $\mathrm{P}$ else $\mathrm{Q}$ fi $\mathrm{R}$ is proof equivalent to if $\mathrm{C}$ then $\mathrm{P} \mathrm{R}$ else $\mathrm{Q} \mathrm{R}$ fi

Proof Any Hoare proof of the former must have steps such as 
$\mathrm{A}$ if $\mathrm{C}$ then $\mathrm{P}$ else $\mathrm{Q}$ fi $\mathrm{D}$

if $\mathrm{C}$ then $\mathrm{P}$ else $\mathrm{Q}$ fi $\mathrm{R}$ B

The final two inferences can equivalently be replaced by

$\mathrm{A} \wedge \mathrm{C} \mathrm{P} R \mathrm{D}$

$\mathrm{A} \wedge \mathrm{C} Q \mathrm{Q} D$

A if $\mathrm{C}$ then $\mathrm{P} R$ else $\mathrm{Q} R$ fi $\mathrm{D}$

The converse implication is shown similarly.

\subsection{Standard Proof-Theoretic Definitions}

Some standard proof-theoretic phrases are now defined.

Definition: a sub-formula of a predicate A is any (well-formed) formula obtained by any number of applications of the following transformations: 1) replace $\neg \mathrm{A}$ by $A ; 2)$ replace $A$ op $B$ by $A$, for any binary boolean operator op $(\vee, \wedge, \Rightarrow$, and so on); or 3) replace A op B by B, for any binary boolean operator op as above.

Thus the four sub-formulas of $\mathrm{C} \wedge \neg \mathrm{D}$ are $\mathrm{C} \wedge \mathrm{D}, \mathrm{C}, \neg \mathrm{D}$, and $\mathrm{D}$, assuming both $\mathrm{C}$ and $\mathrm{D}$ are atomic formulas. Note that being a sub-formula is a transitive relation.

Definition: the logical matrix of a predicate A is the syntax tree showing the structure of its Boolean operators, but ignoring the atomic formulas at the leaves. For example both $\mathrm{B} \wedge(\mathrm{C} \vee \mathrm{D})$ and $x=y \wedge(z>2 \vee \forall t \bullet x(t)>0)$ have the same logical matrix, $\_\wedge\left(-\vee{ }_{-}\right)$.

Definition: suppose $\mathrm{A}=\alpha(\mathrm{B})$ for some substitution $\alpha$. Then $\mathrm{A}$ is said to be an instance or a specialisation of $\mathrm{B}$. B is a generalisation of or more general than A. Note that being an instance is a transitive relation. Also note that the sub-formula and instance relations commute (an instance of a sub-formula is a sub-formula of an instance, and vice versa).

Definition: also, a renaming is an invertible substitution, or one that maps variables to variables.

\section{Equalisation}

Our basic strategy is to search for programs which use sub-formulas of the given verification conditions as the conditional or loop tests. There are, of course, only finitely many sub-formulas of a given formula. There are, however, infinitely many instances. To limit the search space, we need a way to limit the amount of substitution required.

Let us assume therefore that predicates are ordered by i) the substitution ordering $(\mathrm{A} \leq \alpha(\mathrm{A}))$ or else ii) lexicographically in the case of predicates which differ only by a renaming. Note that this ordering is well-founded, so that no decreasing chain of predicates can exist. 


\subsection{Equalising Substitutions}

Definition: $m g c i(\mathrm{~V})$ gives the most general common instance of a set of conditions $\mathrm{V}$, where such an instance exists. Thus $\mathrm{A} \leq \operatorname{mgci}(\mathrm{A}, \mathrm{B}), \mathrm{B} \leq \operatorname{mgci}(\mathrm{A}, \mathrm{B})$, and $\operatorname{mgci}(\mathrm{A}, \mathrm{B}) \leq \mathrm{C}$ for any $\mathrm{C}$ such that $\mathrm{A} \leq \mathrm{C}$ and $\mathrm{B} \leq \mathrm{C}$.

Definition: mges(A, B) gives the most general equalising substitutions to apply to a pair of conditions $\mathrm{A}, \mathrm{B}$ which result in $\operatorname{mgci}(\mathrm{A}, \mathrm{B})$ where this exists. For example if mges $(\mathrm{A}, \mathrm{B})=(\alpha, \beta)$ then $\alpha(\mathrm{A})=\beta(\mathrm{B})=\operatorname{mgci}(\mathrm{A}, \mathrm{B})$.

These definitions are closely related to the notion of unifiers and unification in logic programming. The main difference is that unification applies a single substitution to a set of conditions (or more typically terms) to give a single common instance. A single substitution is a natural notion in the context of declarative programming languages, which are referentially transparent. Imperative languages are not referentially transparent, so it is not surprising that multiple equalising substitutions are required.

The relationship between these notions is as follows. To find the most general common instance of a set of conditions $\mathrm{V}$, first rename the variables in each condition that is a member of $\mathrm{V}$ so that each has a disjoint set of free variables, then unify. That is to say, suppose rename $(\mathrm{V})$ is a function that systematically applies renaming substitutions to elements of $\mathrm{V}$, and returns the resulting, nameclash-free, conditions. Then $\operatorname{mgci}(\mathrm{V})=\operatorname{mgu}($ rename $(\mathrm{V}))$. We can therefore deduce that a most general common instance exists if and only if some common instance does, and that there is an algorithm (based on Robinsons unification algorithm [29]) to find the most general common instance together with the associated substitutions.

Definition: $\operatorname{cms}(\mathrm{V})$ is the set of conditions arising from $\mathrm{V}$ through a finite number of applications of the mgci function and the sub-formula relation, i.e. the closure of $m g c i$ and sub-formula. That is to say, $c m s(\mathrm{~V})$ is the least condition set $\mathrm{W}$ such that a) $\mathrm{V} \subseteq \mathrm{W}$, b) if $\mathrm{W}^{\prime} \subseteq \mathrm{W}$, then $\operatorname{mgci}\left(\mathrm{W}^{\prime}\right) \in \mathrm{W}$, and c) if $\mathrm{A} \in$ $\mathrm{W}$ and $\mathrm{B}$ is a sub-formula of $\mathrm{A}$, then $\mathrm{B} \in \mathrm{W}$.

Lemma 4 (cms) Given a finite condition set V, $\mathrm{cms}(\mathrm{V})$ is also finite. Alternatively, cms is finitary. Remark: Individually, mgci and the sub-formula relation are both finitary. It is not immediately obvious that their union is also.

Proof It is well known that a closure such as cms can be constructed by iterating an appropriate set of generating operations. In this case, appropriate generators are $\mathrm{M}$ and $\mathrm{S}$ where $\mathrm{M}(\mathrm{V})=\{\operatorname{mgci}(\mathrm{W}) \mid\{\} \neq \mathrm{W} \subseteq \mathrm{V}\}$ and $\mathrm{S}(\mathrm{V})=\{\mathrm{A} \mid \mathrm{A}$ subformula $\mathrm{B}$ for some $\mathrm{B} \in \mathrm{V}\}$. Both $\mathrm{M}$ and $\mathrm{S}$ are idempotent (so that, for example, $\mathrm{M}(\mathrm{M}(\mathrm{V}))=\mathrm{M}(\mathrm{V}))$ thanks to related properties of sub-formula and mgci so we need only consider alternating sequences of the $\mathrm{M}$ and $\mathrm{S}$ generators. Finally note that, taking a sub-formula reduces the boolean matrix of the original formula, whereas any instance, and in particular any mgci, has the same matrix, and thus the same number of boolean operators, as the original formula(s). The closure $\mathrm{cms}(\mathrm{V})$ is therefore constructed by alternating sequences of $\mathrm{M}$ and $\mathrm{S}$ generators at most $\# \mathrm{~V}$ long, where $\# \mathrm{~V}$ is the maximum boolean operator count of any formula in V. Since mgci and sub-formula are both finitary, and the union of a finite number of finite sets is finite, this proves cms is finitary. 


\subsection{Equalising Programs}

We now come to the important idea of an equalising program, or EP for short. This, simplistically, is one in which the only assignments are ones which equalise their post-condition with that of some other assignment. A precise definition follows below.

It is convenient at this point to introduce the idea of assertions and asserted programs.

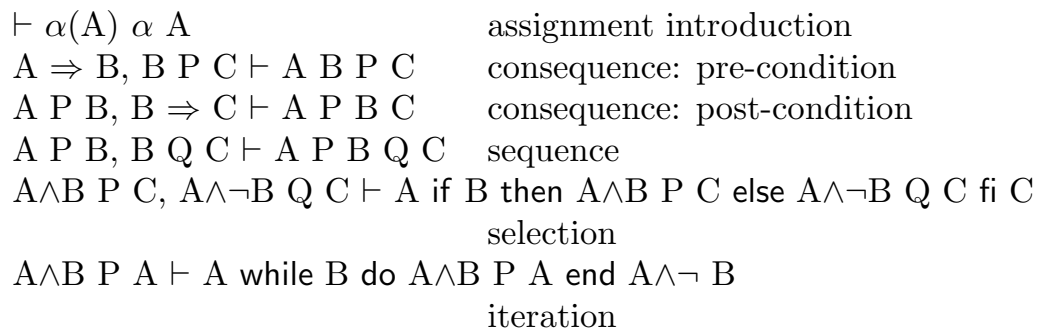

Figure 2: Asserted Hoare Logic

Definition: A fully asserted program is one which is generated by the following axiom and inference rules, which are the same as in traditional Hoare logic, but incorporating an extra assertion or assertions in the consequent of each law.

Figure 2 (overleaf) presents asserted Hoare logic as a formal inference system. Definition: an asserted program (a.k.a. a partially asserted program) is one which can be obtained from a fully asserted program by deleting some of the assertions.

In what follows, asserted programs will be used to simplify the presentation. Observe that a fully asserted program is in some sense equivalent to a Hoare proof. In particular, every Hoare proof can be converted to a fully asserted program and vice versa [31] (chapter 15).

Definition: an equalising proof is a Hoare proof, of a triple A P B say and based on verification conditions $\mathrm{V}$, in which every assignment introduction is of the form $\mathrm{C} \alpha \mathrm{D}$ where either $\mathrm{C} \in \mathrm{cms}(\mathrm{V}, \mathrm{B})$ or $\mathrm{C}=\mathrm{C}_{1} \wedge \mathrm{C}_{2}$, where $\mathrm{C}_{1} \wedge \mathrm{C}_{2} \in$ $\operatorname{cms}(\mathrm{V}, \mathrm{B})$ and similarly for D.

Definition: an equalising triple is one which has an equalising proof, and similarly an equalising program.

Here is a contrived example to show why the post-condition B is required as an argument to $\mathrm{cms}$ in the definition above:

$\mathrm{C}(f(x))$ if $\mathrm{D}(g(y))$ then $y:=g(y)$ else $x:=f(x)$ end $\mathrm{C}(f(x)) \wedge \mathrm{D}(y)$

with verification condition $\mathrm{C}(x) \wedge \mathrm{D}(g(y)) \Rightarrow \mathrm{C}(f(x)) / \mathrm{D}(y)$

The condition $\mathrm{D}(g(y))$ arises through equalising the post-condition $\mathrm{C}(f(x)) \wedge$ $\mathrm{D}(y)$ with the antecedent of the verification condition $\mathrm{C}(x) \wedge \mathrm{D}(g(y))$ and taking the final sub-formula.

Lemma 4 (corollary) It follows from lemmas 4 and this definition that it is decidable whether an equalising program $\mathrm{P}$ exists, based on a given set of verification conditions $\mathrm{V}$, satisfying given pre- and post-conditions $\mathrm{A}$ and $\mathrm{B}$. 
A simple algorithm would be to search through the space of all proofs up to certain length. This is sufficient as $c m s(\mathrm{~V}, \mathrm{~B})$ is finite by lemma 4 , and by lemma 2 (substitutability) at most one sub-program satisfying each pair of preand post-conditions $\mathrm{C}, \mathrm{D} \in \operatorname{cms}(\mathrm{V}, \mathrm{B})$ is required. Only proofs of length up to $\mathrm{N}^{2}$ need be considered, therefore, where $\mathrm{N}=\# \mathrm{cms}(\mathrm{V}, \mathrm{B})$. More efficient algorithms than this can no doubt be devised, but this paper is concerend with existence, not efficiency.

A useful property of equalising programs is given in the following lemma.

Lemma 5 If A P B and B Q C are both equalising triples, so too is A P Q C. Proof Combining the proofs of the two given equalising triples with a final inference using the law of sequence gives a proof of A P Q C. In this proof, every conclusion has pre- and post-conditions occurring in, or constructed from, $\operatorname{cms}(\mathrm{V}, \mathrm{B})$ or $\mathrm{cms}(\mathrm{V}, \mathrm{C})$. Moreover $\mathrm{B}$, which is the initial pre-condition of B Q $\mathrm{C}$, must occur in or be constructed from $\mathrm{cms}(\mathrm{V}, \mathrm{C})$. By closure therefore, the pre- and post-conditions of every conclusion occur in or are constructed from $\operatorname{cms}(\mathrm{V}, \mathrm{C})$ as required.

Definition: an $\alpha$-equalising proof is the proof corresponding the asserted program $\alpha(\mathrm{A}) \alpha \mathrm{A}$ P B for some assignment statement $\alpha$. That is to say, it consists of an equalising proof, of A P B say, followed by two further steps of the form

$\alpha(\mathrm{A}) \alpha \mathrm{A} \quad$ assignment axiom

$\alpha(\mathrm{A}) \alpha \mathrm{P} \mathrm{C}$ sequence

Similarly for $\alpha$-equalising triples and $\alpha$-equalising programs.

The decision procedure for the existence of an equalising program can easily be extended to $\alpha$-equalising programs by checking which, if any, conditions in $\operatorname{cms}(\mathrm{V}, \mathrm{B})$ are generalisations of the given pre-condition $\mathrm{A}$, and determining if it is possible to establish post-condition B from any of these.

In the following $\alpha$-equalising programs provide a "normal form" for Hoare proofs.

\section{The Main Theorem}

\subsection{Inductive Lemmas}

This leads us to our main result: if A P B is a valid Hoare triple based on verification conditions $\mathrm{V}$, then for $\alpha$ and $\mathrm{P}^{\prime}, \mathrm{A} \alpha \mathrm{P}^{\prime} \mathrm{B}$ is a $\alpha$-equalising Hoare triple based on $\mathrm{V}$. The proof of this is by induction, and to simplify the presentation, the main steps are given in the following lemmas, which demonstrate the ability to transform an equalising program following by an assignment statement into an assignment following by an equalising program, i.e. an $\alpha$-equalising program. The induction is on the depth and size of the program P. Note that the more elegant technique of structural induction cannot be applied here because of the transformations used to prove lemma 8 below.

Definition: the depth of a program $\mathrm{P}$ is the greatest level of nesting of any statement in $\mathrm{P}$. To be precise 


$$
\begin{array}{ll}
\operatorname{depth}(\alpha)=0 & \text { assignment } \\
\operatorname{depth}(\mathrm{P} \mathrm{Q})=\max (\operatorname{depth}(\mathrm{P}), \operatorname{depth}(\mathrm{Q})) & \text { sequence } \\
\operatorname{depth}(\text { while } \mathrm{A} \text { do } \mathrm{P} \text { end })=\operatorname{depth}(\mathrm{P})+1 & \text { iteration } \\
\operatorname{depth}(\text { if } \mathrm{A} \text { then } \mathrm{P} \text { else } \mathrm{Q} \text { fi })=\max (\operatorname{depth}(\mathrm{P}), \operatorname{depth}(\mathrm{Q}))+1 & \text { conditional }
\end{array}
$$

Definition: The size of a program is found by counting the number of leaves in its syntax tree, i.e. the number of assignment statements it contains.

Lemma 6 (base case) Suppose A P $\alpha$ B is a valid Hoare triple based on verification conditions $\mathrm{V}$ where program $\mathrm{P}$ has depth 0 , then there exist $\alpha^{\prime}, \mathrm{P}^{\prime}$ such that $\mathrm{A} \alpha^{\prime} \mathrm{P}^{\prime} \mathrm{B}$ is a valid $\alpha$-equalising triple also based on $\mathrm{V}$.

Proof Note that a program of depth 0 can have no conditionals nor loops. The derivation of $\mathrm{P} \alpha$ therefore can involve only the assignment axiom and the laws of sequence and consequence. Any sequence of assignments without intervening consequence can be merged into a single multiple assignment [23] so $\mathrm{P} \alpha$ can equivalently be presented in a form whereby every pre-condition, except possibly the first, is the antecedent of a verification condition, and similarly every postcondition, except possibly the last, is the consequent of a verification condition. This, by definition, is an $\alpha$-equalising proof.

The following two lemmas both include as one of their assumptions the induction hypothesis used in the proof of the main theorem below. This hypothesis states that given any program $\mathrm{Q}$ such that $\operatorname{depth}(\mathrm{Q})<\operatorname{depth}(\mathrm{P})$, or with the same depth as $\mathrm{P}$ but smaller in size, and conditions $\mathrm{A}, \mathrm{B}$ such that $\mathrm{A} \mathrm{Q} \mathrm{B}$ is a valid Hoare triple based on verification conditions $\mathrm{V}$, then there exist $\beta, \mathrm{R}$ such that $\mathrm{A} \beta \mathrm{R} \mathrm{B}$ is a valid $\alpha$-equalising triple also based on $\mathrm{V}$, and with the same depth as Q.

Lemma 7 (conditional) Suppose A P $\alpha$ B is a valid Hoare triple based on verification conditions $\mathrm{V}$ where $\mathrm{P}$ is a conditional, and the induction hypothesis holds. Then there exist $\alpha^{\prime}, \mathrm{P}^{\prime}$ where $\mathrm{P}^{\prime}$ has the same depth as $\mathrm{P}$ such that $\mathrm{A}$ $\alpha^{\prime} \mathrm{P}^{\prime} \mathrm{B}$ is a valid $\alpha$-equalising triple also based on $\mathrm{V}$.

Proof By lemma 3, the trailing assignment $\alpha$ can be moved into the two legs of the conditional statement. This does not affect their depth. The induction hypothesis therefore applies to both the then- and the else-parts of the conditional, which therefore have equivalent $\alpha$-equalising forms. This leads to a valid Hoare triple of the form $\mathrm{A}$ if $\mathrm{B}$ then $\mathrm{A} \wedge \mathrm{B} \alpha_{1} \mathrm{~A}_{1} \wedge \mathrm{B}_{1} \mathrm{P}_{1} \mathrm{C}$ else $\mathrm{A} \wedge \neg \mathrm{B} \alpha_{2} \mathrm{~A}_{2} \wedge \neg \mathrm{B}_{2} \mathrm{P}_{2}$ $C$ fi $C$, based on $V$, where $A_{1} \wedge B_{1} P_{1} C$ and $A_{2} \wedge B_{2} P_{2} C$ are equalising. Now let $\mathrm{A}^{\prime} \wedge \mathrm{B}^{\prime}=\operatorname{mgci}\left(\mathrm{A}_{1} \wedge \mathrm{B}_{1}, \mathrm{~A}_{2} \wedge \mathrm{B}_{2}\right),\left(\alpha_{1}^{\prime}, \alpha_{2}^{\prime}\right)=\operatorname{mges}\left(\mathrm{A}_{1} \wedge \mathrm{B}_{1}, \mathrm{~A}_{2} \wedge \mathrm{B}_{2}\right)$, and $\beta$ be such that $\mathrm{A} \wedge \mathrm{B}=\beta\left(\mathrm{A}^{\prime} \wedge \mathrm{B}^{\prime}\right)$. Now $\mathrm{A} \beta \mathrm{A}^{\prime}$ if $\mathrm{B}^{\prime}$ then $\mathrm{A}^{\prime} \wedge \mathrm{B}^{\prime} \alpha_{1}^{\prime} \mathrm{A}_{1} \wedge \mathrm{B}_{1} \mathrm{P}_{1} \mathrm{C}$ else $\mathrm{A}^{\prime} \wedge \neg \mathrm{B}^{\prime} \alpha_{2}^{\prime} \mathrm{A}_{2} \wedge \neg \mathrm{B}_{2} \mathrm{P}_{2} \mathrm{C}$ fi $\mathrm{C}$ is an $\alpha$-equalising triple as required.

Lemma 8 (loop) Suppose A P $\alpha$ B is a valid Hoare triple based on verification conditions $\mathrm{V}$ where $\mathrm{P}$ is a loop, and the induction hypothesis holds. Then there exist $\alpha^{\prime}, \mathrm{P}^{\prime}$ where $\mathrm{P}^{\prime}$ has the same depth as $\mathrm{P}$ such that $\mathrm{A} \alpha^{\prime} \mathrm{P}^{\prime} \mathrm{B}$ is a valid $\alpha$-equalising triple also based on $\mathrm{V}$.

Proof The given Hoare triple can be assumed to of the form $\mathrm{A}$ while $\mathrm{B}$ do $\mathrm{A} \wedge \mathrm{B} \beta$ $\mathrm{A}_{1} \wedge \mathrm{B}_{1} \mathrm{P} \mathrm{A}$ end $\mathrm{A} \wedge \neg \mathrm{B} \alpha \mathrm{A}_{2} \wedge \neg \mathrm{B}_{2}$, say. (There is no loss of generality here since $\beta$ could be the empty assignment, skip.) Now let $\mathrm{A}^{\prime} \wedge \mathrm{B}^{\prime}=\operatorname{mgci}\left(\mathrm{A}_{1} \wedge \mathrm{B}_{1}, \mathrm{~A}_{2} \wedge \mathrm{B}_{2}\right)$, $\left(\beta^{\prime}, \alpha^{\prime}\right)=\operatorname{mges}\left(\mathrm{A}_{1} \wedge \mathrm{B}_{1}, \mathrm{~A}_{2} \wedge \mathrm{B}_{2}\right)$, and $\gamma_{1}$ be such that $\mathrm{A} \wedge \mathrm{B}=\gamma_{1}\left(\mathrm{~A}^{\prime} \wedge \mathrm{B}^{\prime}\right)$. Now 
A $\gamma_{1} \mathrm{~A}^{\prime}$ while $\mathrm{B}^{\prime}$ do $\mathrm{A}^{\prime} \wedge \mathrm{B}^{\prime} \beta^{\prime} \mathrm{A}_{1} \wedge \mathrm{B}_{1} \mathrm{P}$ A $\gamma_{1} \mathrm{~A}^{\prime}$ end $\mathrm{A}^{\prime} \wedge \neg \mathrm{B}^{\prime} \quad \alpha^{\prime} \quad \mathrm{A}_{2} \wedge \neg \mathrm{B}_{2}$ is a valid Hoare triple. By the induction hypothesis, the valid triple $A_{1} \wedge B_{1} P$ A $\gamma_{1} \mathrm{~A}^{\prime}$ has an $\alpha$-equalising form, $\mathrm{A}_{1} \wedge \mathrm{B}_{1} \delta \mathrm{P}_{1} \mathrm{~A}^{\prime}$ say. Substituting this in the previous triple gives $\mathrm{A} \gamma_{1} \mathrm{~A}^{\prime}$ while $\mathrm{B}^{\prime}$ do $\mathrm{A}^{\prime} \wedge \mathrm{B}^{\prime} \beta^{\prime} \mathrm{A}_{1} \wedge \mathrm{B}_{1} \delta \mathrm{P}_{1} \mathrm{~A}^{\prime}$ end $\mathrm{A}^{\prime} \wedge \neg \mathrm{B}^{\prime} \quad \alpha^{\prime} \quad \mathrm{A}_{2} \wedge \neg \mathrm{B}_{2}$. The sequence of assignments $\beta^{\prime} \delta$ can be merged to a single assignment $\beta_{1}$ say (dropping the intermediate assertion $A_{1} \wedge B_{1}$ ). Finally, therefore, we have the valid triple $\mathrm{A} \gamma_{1} \mathrm{~A}^{\prime}$ while $\mathrm{B}^{\prime}$ do $\mathrm{A}^{\prime} \wedge \mathrm{B}^{\prime} \beta_{1} \mathrm{P}_{1} \mathrm{~A}^{\prime}$ end $\mathrm{A}^{\prime} \wedge \neg \mathrm{B}^{\prime} \alpha^{\prime} \mathrm{A}_{2} \wedge \neg \mathrm{B}_{2}$, which is in the same form as the original program. The same sequence of steps can therefore be applied repeatedly to give a sequence of such programs. Note that in this sequence of programs, the pre-condition of the loop body is in each case a generalisation of the previous pre- condition. The sequence of these pre-conditions, $\mathrm{A} \wedge \mathrm{B}, \mathrm{A}^{\prime} \wedge \mathrm{B}^{\prime}, \mathrm{A}^{\prime \prime} \wedge \mathrm{B}^{\prime \prime}$, and so on cannot continue forever strictly decreasing, by well-foundedness of the generalisation relation. This means that at some point the pre-condition will be the most common instance of the post-conditions, making the loop body equalising, and the whole program $\alpha$-equalising as required (the leading assignments $\gamma_{1}, \gamma_{2}, \gamma_{3}$, ... having been merged into a single assignment).

\subsection{Proof of the Main Theorem}

Theorem 1 Suppose A P B is a valid Hoare triple based on verification conditions $\mathrm{V}$. Then for some $\alpha$ and $\mathrm{P}^{\prime}, \mathrm{A} \alpha \mathrm{P}^{\prime} \mathrm{B}$ is a valid $\alpha$-equalising triple based on $\mathrm{V}$.

Proof By induction the depth and size of P.

Base case $(\operatorname{depth}(\mathrm{P})=0)$ : the theorem follows from lemma 6 .

Induction step $(\operatorname{depth}(\mathrm{P})>0)$ : the induction hypothesis is that the theorem holds for any program $\mathrm{Q}$ whose depth is strictly less than that of $\mathrm{P}$, or any program $\mathrm{Q}$ whose depth is the same as that of $\mathrm{P}$ but whose size is small than that of $\mathrm{P}$. To prove the induction step, consider the structure of $\mathrm{P}$.

Case 1 ( $\mathrm{P}$ is a conditional)

The theorem follows from the induction hypothesis and lemma 7 .

Case 2 ( $\mathrm{P}$ is a loop)

The theorem follows from the induction hypothesis and lemma 8 .

Case 3 ( $\mathrm{P}$ is a sequential composition)

By the induction hypothesis, the theorem holds for both sub-programs of $\mathrm{P}$, which gives rise to the triple $\mathrm{A} \alpha_{1} \mathrm{P}_{1} \alpha_{2} \mathrm{P}_{2} \mathrm{~B}$, where each $\alpha_{i} \mathrm{P}_{i}$ is $\alpha$-equalising. This sequence can be re-associated using lemma 1 to give $\mathrm{A} \alpha_{1}\left(\mathrm{P}_{1} \alpha_{2}\right) \mathrm{P}_{2} \mathrm{~B}$ where $\mathrm{P}_{1}$ is equalising. Without loss of generality $\mathrm{P}_{1}$ is either an assignment, a conditional, or a loop. By lemmas 6, 7 and 8, and since $\mathrm{P}_{1} \alpha_{2}$ ) is smaller in size and no deeper then the original program, there is an $\alpha$-equalising $\alpha_{3} \mathrm{P}_{3}$, say, with the same pre- and post-condition that can be substituted in to give the valid triple $\mathrm{A} \alpha_{1} \alpha_{3} \mathrm{P}_{3} \mathrm{P}_{2} \mathrm{~B}$. The leading assignments here, $\alpha_{1} \alpha_{3}$, can be merged to give the required $\alpha$-equalising triple. 


\subsection{Total Correctness Logic and the Main Theorem}

Next we briefly show the same result holds for a total correctness Hoare Logic. Consider a Hoare logic for total correctness, with an iteration law that ensures termination and not just preservation of the invariant. For example, the classic iteration law

$\mathrm{A} \wedge \mathrm{B} \mathrm{P} \mathrm{A} \vdash \mathrm{A}$ while $\mathrm{B}$ do $\mathrm{P}$ end $\mathrm{A} \wedge \neg \mathrm{B}$

could be replaced by an extended law

$\mathrm{A} \wedge \mathrm{B} \wedge \mathrm{X}=E \mathrm{P} \quad \mathrm{A} \wedge 0 \leq E<\mathrm{X} \vdash \mathrm{A}$ while $\mathrm{B}$ do $\mathrm{P}$ end $\mathrm{A} \wedge \neg \mathrm{B}$

where $\mathrm{X}$ is a logical constant that does not occur free in $\mathrm{A}$, nor in $\mathrm{B}$, and $E$ is an integer-valued expression. (There are of course many possible loop laws. This particularly simple formulation, which is inspired by that of Morgan [27], is amenable to proof-theoretic techniques. Of course, any well-founded relation could occur here, not just natural number ordering.)

A logical constant is a special kind of variable. As with other kinds, it is assumed an infinite number are available for use traditionally these are represented by giving them upper case names, or zero-subscripting. It is allowed to occur in logical conditions, but not in the program text. This fact has important consequences. Firstly, X can never be the target of an assignment, so it cannot be replaced via the assignment axiom. Secondly, X can never occur in the condition of a loop or conditional statement, so it cannot disappear as a result of applying the laws of iteration or selection.

Lemma 9 In a total correctness proof, any intermediate Hoare triples ending with the loop post- condition $\mathrm{A} \wedge 0 \leq E<\mathrm{X}$, and proved using the assignment axiom, and laws of sequence, selection, and iteration, must have as its precondition $\alpha(\mathrm{A}) \wedge 0 \leq \alpha(E)<\mathrm{X}$.

Proof By induction on the (size of the) proof.

A similar result holds for the loop pre-condition.

Lemma 10 In a total correctness proof, any intermediate Hoare triples ending in the loop pre- condition $\mathrm{A} \wedge \mathrm{B} \wedge \mathrm{X}=E$, and proved using assignment axiom, and laws of sequence, selection, and iteration, must have as its post-condition $\beta^{-1}(\mathrm{~A}) \wedge \mathrm{X}=\beta^{-1}(E)$, for some assignment $\beta$. (Note that the use of the inverse substitution $\beta^{-1}$ means that the post-condition here is a generalisation of the pre-condition.)

Proof By induction on the (size of the) proof.

Combining these two results, we see that conditions $\alpha(\mathrm{A}) \wedge 0 \leq \alpha(E)<\mathrm{X}$ and $\beta^{-1}(\mathrm{~A}) \wedge \mathrm{X}=\beta^{-1}(E)$ must occur as the consequence and antecedent in some verification condition, before the hypothesis $\mathrm{A} \wedge \mathrm{B} \wedge \mathrm{X}=E \mathrm{P} A \wedge 0 \leq E<\mathrm{X}$ of the new loop law can be derived.

Matching $\alpha(\mathrm{E})$ and $\beta^{-1}(E)$ gives a finite number of possibilities for expression $E$, up to renaming. Cycling through all possible variables (the ones that occur in the closure $c m s(\mathrm{~V}, \mathrm{~B}))$ gives a finite number of possibilities for expression $E$. Combining this with the techniques used for the partial correctness theorem gives an equivalent decision procedure for the total correctness variant of Hoare logic presented here - since the new iteration law contains the original one as a sub-formula, the technique of equalising still applies, although the 
actual definitions and lemmas used must change to match the form of the new iteration law.

\section{Discussion}

\subsection{Verification Conditions for Total Correctness}

In this section, the integer square root case study introduce earlier is considered again, with a view to indicating how verification conditions can be discovered without first having to write the code. Also, the conditions given here are for total correctness.

Following Gries' strategy for developing a loop [16]: "first develop the guard $\mathrm{B}$ so that $\mathrm{A} \wedge \mathrm{B} \Rightarrow \mathrm{C}$ " it is clear to see how the invariant and guard can be determined from the following theorem:

$$
r^{2} \leq s<q^{2} \wedge \neg(r+1 \neq q) \Rightarrow r^{2} \leq s<(r+1)^{2}
$$

The following condition indicates how this invariant can be established:

$$
s \geq 0 \Rightarrow 0^{2} \leq s<(s+1)^{2}
$$

Now consider a possible variant: $q-r$. One way to decrease this quantity is by finding a value between $q$ and $r$. The feasibility of doing so is confirmed by the following theorem:

$$
r^{2} \leq s<q^{2} \wedge r+1 \neq q \Rightarrow r<(q+r) \div 2<q
$$

Finally note that a value between $\mathrm{r}$ and $\mathrm{q}$ can be used to re-establish the invariant and decrease the variant:

$$
\begin{aligned}
& r^{2} \leq s<q^{2} \wedge r<p<q \wedge s<p^{2} \Rightarrow 0 \leq p-r<q-r \\
& r^{2} \leq s<q^{2} \wedge r<p<q \wedge \neg\left(s<p^{2}\right) \Rightarrow 0 \leq q-p<q-r
\end{aligned}
$$

For book-keeping reasons these last three conditions must in fact be recorded more verbosely. The first one, for example, should not be

$$
r^{2} \leq s<q^{2} \wedge r+1 \neq q \Rightarrow r<(q+r) \div 2<q
$$

but rather

$$
\begin{aligned}
& r^{2} \leq s<q^{2} \wedge r+1 \neq q \wedge \mathrm{X}=q-r \Rightarrow \\
& r^{2} \leq s<q^{2} \wedge r<(q+r) \div 2<q \wedge \mathrm{X}=q-r
\end{aligned}
$$

The second one should not be

$$
r^{2} \leq s<q^{2} \wedge r<p<q \wedge s<p^{2} \Rightarrow 0 \leq p-r<q-r
$$

but rather

$$
\begin{aligned}
& r^{2} \leq s<q^{2} \wedge r<p<q \wedge \mathrm{X}=q-r \wedge s<p^{2} \Rightarrow \\
& r^{2} \leq s<p^{2} \wedge 0 \leq p-r<\mathrm{X}
\end{aligned}
$$

and similarly for the third condition.

Adding the extra constraints such as $\mathrm{X}=q-r$ means these conditions syntactically match the inference laws of Hoare logic, even though the extra clauses add no logical content.

This case study is based on Chapter 8 of Morgan's textbook on refinement [27] (see also the second edition, 1994). The development presented in that book (figure 19.2) has 11 refinement steps which use 7 different refinement laws and depend on 5 verification conditions, which are essentially the same as the ones given here. Morgan does not explicitly list these conditions, but notes that the initial development contained two errors, one a transcription error, the 
other a logical error. He then makes the observation that "mathematical rigour cannot eliminate mistakes entirely: nevertheless it does reduce their likelihood dramatically".

Gries [16] gives as one of his main principles: "a program and its proof should be developed hand-in-hand, with the proof usually leading the way". Gries also has other useful heuristics, such as "four ways of weakening a predicate", by "deleting a conjunct, replacing a constant by a variable, enlarging the range of a variable, or by adding a disjunct". Other heuristics however are oriented toward programs, not predicates. (For example, "develop one leg of a conditional by finding program $\mathrm{P}$ such that $\mathrm{A} \Rightarrow w p(\mathrm{P}, \mathrm{B}) "$.)

For a development methodology focussed exclusively on predicates, see work of Hehner [17, 18, 19].

\subsection{Formal Verification Tools}

Early criticism of formal program verification focussed on the likelihood of human errors, and the fallibility of social processes such as peer review [12], [13]. It is clear that to increase confidence in programs (cf. algorithms), high quality automated tool support is required for processes such as theorem proving, proof checking, and code generation.

Broadly speaking, three kinds of formal verification tools have been proposed.

Firstly there are mathematically-based toolkits, such as GYPSY [2], Atelier B [6] and Perfect Developer [11]. Programs are written using mathematically defined programming notations and formal specifications or annotations. Conditions are generated from this input, which are must then be verified using interactive or automated theorem proving systems. Similar verification condition generation (VCG) systems have also been implemented that use conventional programming languages but with formal annotations, for example the Java Modelling Language JML [26], and Spec\# [30]

A second approach is represented by the refinement calculator [7]: a finegrained interactive system for program refinement. Some of these refinement steps will generate verification conditions that must be discharged by a theorem prover.

Finally, the logic compiler: Hehners vision is of "compilers, with built-in theorem provers" which will be able to "point out the location of a logic error the same way as they do now with a syntax error" [18]. To date this vision has not been realised.

It is worthwhile comparing the VCG approach with the logic compiler approach. The latter has the advantage of being proof-driven, whereby developers verify theorems before the code is developed. Indeed, since code is generated from the theorems, development is necessarily verification first. With the VCG approach, errors may discovered days or weeks after they are introduced, when it is finally realised that some verification condition cannot be proved because it is in fact false. A proof-driven approach would discover this fact sooner. A 
collection of theorems is built up, then fed to an executable code generator, for example a more efficient version of the algorithm described in this paper.

The problem that can now arise is that the supplied theorems are insufficient, preventing the derivation and output of a (correct-by-construction) program. It is crucial that in this case the "logic compiler" does not simply fail, but provides helpful diagnostics so that the developer can decide what additional theorems must be proved and supplied as additional input to the compiler. More useful than this, in fact, would be for the compiler itself to postulate these additional theorems and pass them directly to the automated proof system: rather than requiring a complete set of verification conditions, just a partial set should suffice, or conditions that are logically equivalent but not necessarily in the exact syntactic form. As a trivial example of this, it would be helpful if the logic compiler would accept a logically equivalent formulation, for example $\mathrm{B} \wedge \mathrm{A} \Rightarrow \mathrm{C}$ instead of $\mathrm{A} \wedge \mathrm{B} \Rightarrow \mathrm{C}$, but also including more sophisticated logical transformations, as well as addition of the "book-keeping" constraints noted above.

A further point is that there is typically a certain amount of redundancy in the verification conditions required by Hoare logic. In the case study above, for example, each clause occurs on average about twice, ignoring renaming. This redundancy could be avoided by introducing local definitions, or by careful insertion of additional clauses by the logic compiler using appropriate heuristics.

Clearly, these extra features of the logic compiler would evolve through practice and experience of actual use of such a tool.

\subsection{Related and Future Work}

Hoare logic has been extensively studied since it was first published [20]. Completeness [9] and incompleteness [8] results have been published, as has an extensive survey [3].

An earlier version of the main result given here was presented at a workshop [14]. This was based on a specially constructed program logic, not classical Hoare Logic.

The work here differs from previous approaches to predicative programming $[17,19,24]$ in that the latter give a first order or predicative semantics to programs, with $\Leftarrow$ as program refinement. Although predicative semantics is quite natural for finite programs, but special care is needed with iteration and recursion to give satisfactory results. The approach presented here is neutral with respect to semantics, since it is proof-theoretic. As with any axiomatic approach, no model for iteration is provided, only an inference law.

Constructive, or intuitionist, logic provides an alternative way to generating programs from proofs [25] which has been implemented in systems such as NUPRL [10] among others. The approach presented here is neutral with respect to the underlying logic, which can be either classical or constructive. A further difference is that here programs are generated from the verification conditions, not their proofs. 
The result given here has been shown to apply to two versions of Hoare logic. It would be interesting to know whether it holds for larger, more realistic systems such as the Hoare Logic for Pascal [22] or the Refinement Calculus [27]. The preceding section described automated support for predicative programming based on this result, which has yet to be implemented.

\section{References}

[1] Abrial JR: The B Book, Cambridge, 1996.

[2] Ambler AL et al: GYPSY: A Language for Specification and Implementation of Verifiable Programs, ACM SIGPLAN Notices 12: 1-10, March 1977.

[3] Apt KR: Ten years of Hoare's logic, ACM Transactions on Programming Languages and Systems 3: 431-483, 1981.

[4] Back RJR: On the Correctness of Refinement Steps in Program Development, Report A-1978-5, University of Helskinki, 1978.

[5] Back RJR, von Wright J: Refinement Calculus: a systematic introduction, Springer, 1998.

[6] Behm P et al: Meteor: A Successful Application of B in a Large Project, LNCS 1708: 369-387, Springer, 1999.

[7] Butler MJ, Långbacka: Program Derivation Using the Refinement Calculator, TPHOLs: 93-108, 1996.

[8] Clarke EM: Programming Language Constructs for which it is impossible to obtain good Hoare axiom systems, JACM 26(1): 129-147, 1979.

[9] Cook SA: Soundness and Completeness of an Axiom System for Program Verification, SIAM Journal of Computing 7: 70-90, 1978.

[10] Constable RL: Implementing Mathematics with the Nuprl Proof Development System, Prentice Hall, 1986.

[11] Crocker D: Safe Object-Oriented Software, Twelfth Critical Systems Symposium: 19-41, Springer, 2004.

[12] de Millo RA, Lipton RJ, Perlis AJ: Social Processes and Proof of Theorems and Programs, CACM 22(5): 271-280, 1979.

[13] Fetzer JH: Program Verification: the very idea, CACM 31(9): 1048-1063.

[14] Gravell AM: Logical Refinement of Imperative Programs: generating code from verified conditions, (Constraint) Logic Programming and Software Engineering 2000, Gupta G, Ramakrishnan IV (eds.), Imperial College, London, July 2000. 
[15] Gries D: The Multiple Assignment Statement, LNCS 69: 100-112, Springer, 1978.

[16] Gries D: The Science of Programming, Springer, 1981.

[17] Hehner ECR: Predicative Programming: I, Communications of the ACM 27: 134-143, 1984.

[18] Hehner ECR, Gupta LE, Malton AJ: Predicative Methodology, Acta Informatica 23:487-505, 1986.

[19] Hehner ECR: A Practical Theory of Programming, Springer, 1993.

[20] Hoare CAR: An Axiomatic Basis of Computer Programming, Communications of the ACM 12: 576-580, 1969.

[21] Hoare CAR: Procedures and Parameters: An Axiomatic Approach, Symposium on Semantics of Algorithmic Languages, LNCS 188: 102-116, 1971.

[22] Hoare CAR, Wirth N: An Axiomatic Definition of the Programming Language Pascal, Acta Informatica 2:335-355, 1973.

[23] Hoare CAR et al: Laws of Programming, Communications of the ACM 30: 672-686, 1987.

[24] Hoare CAR, He J: Unified Theories of Programming, Prentice Hall, 1998.

[25] Howard WA: The Formulae-as-Types notion of Construction, To H. B. Curry: Essays on Combinatory Logic, Lambda Calculus and Formalism, pages 479-490, 1980.

[26] Leavens GT, Baker AL, Ruby C: JML: a Java Modelling Language, Formal Underpinnings of Java, OOPSLA, 1998.

[27] Morgan CC: Programming from Specifications, Prentice Hall, 1990.

[28] Morris JM: A Theoretical Basis for Stepwise Refinement and the Programming Calculus, Science of Computer Programming 9(3):287-306, 1987.

[29] Robinson JA: Computational Logic: The Unification Computation, Machine Intelligence 6:63-72, 1971.

[30] Rustan K, Leino M, Barnett M, Schulte W: The Spec\# Programming System: an overview, Microsoft Research, 2004

[31] Sperschneider V, Antoniou G: Logic: a foundation for Computer Science, Addison Wesley, 1991. 